\title{
Moiré quantum chemistry: charge transfer in transition metal dichalcogenide superlattices
}

\author{
Yang Zhang* Noah F. Q. Yuan* * $^{*}$ and Liang Fu \\ Department of Physics, Massachusetts Institute of Technology, Cambridge, Massachusetts 02139, USA
}

\begin{abstract}
Transition metal dichalcogenide (TMD) bilayers have recently emerged as a robust and tunable moiré system for studying and designing correlated electron physics. In this work, by combining large-scale first principle calculation and continuum model approach, we provide an electronic structure theory that maps long-period heterobilayer TMD superlattices onto diatomic crystals with cations and anions. We find that the interplay between moiré potential and Coulomb interaction leads to filling-dependent charge transfer between MM and MX regions several nanometers apart. We show that the insulating state at half-filling found in recent experiments on $\mathrm{WSe}_{2} / \mathrm{WS}_{2}$ is a charge-transfer insulator rather than a Mott-Hubbard insulator. Our work reveals the richness of simplicity in moiré quantum chemistry.
\end{abstract}

Following the recent discovery of correlated insulators and unconventional superconductivity in twisted bilayer graphene [1, 2] and trilayer graphene-hBN heterostructure [3, 4, artificial moiré superlattices have emerged as a new venue for realizing and controlling correlated electron phenomena. The moiré superlattices and natural solids differ greatly in the magnitude of characteristic length and energy. In solids, the average distance between electrons is typically comparable to atomic spacing in the order of $\AA$ and their kinetic and interaction energies are typically in the order of $\mathrm{eV}$, while in moiré superlattices a mobile charge is shared by 1000-10000 atoms so that the characteristic length and energy scales are in the order of $10 \mathrm{~nm}$ and $10-100 \mathrm{meV}$ respectively. Correspondingly, the quantum chemistry of natural solids involves complex intra-atomic and long-range interactions, while low-energy charge carriers in moiré superlattices only feel a long-period potential and interact with each other predominantly via the long-range Coulomb repulsion. Therefore, quantum chemistry can be simpler in moiré systems.

In twisted bilayer graphene, the emergence of strong correlation effects requires fine tuning to a magic twist angle, where the moiré energy bands become flattened [5] and sensitive to microscopic details such as lattice relaxation [6-11] and strain [12. On the other hand, transition metal dichalcogenide (TMD) bilayers 13 16 have a much simpler moiré band structure. In TMD heterobilayers such as $\mathrm{WSe}_{2} / \mathrm{WS}_{2}$, the valence moiré bands are simply formed by holes moving in a periodic moiré potential. Therefore, TMD superlattices provide a robust platform to study many-body physics with a highly tunable kinetic energy and local interaction strength.

Very recently, a correlated insulating phase has been observed in $\mathrm{WSe}_{2} / \mathrm{WS}_{2}$ at half-filling [15, 16] of the topmost valence moiré bands with a charge gap around $150 \mathrm{~K}$ $(\sim 10 \mathrm{meV})$, and regarded as a canonical Mott-Hubbard insulator [17. In this scenario, the topmost moiré band

*These two authors contributed equally to this work. is well separated from the rest; its charge distribution is tightly localized near the moiré potential minima, forming a triangular lattice. Strong on-site Coulomb repulsion $U$ suppresses double occupancy and creates an insulating gap at half-filling in the order of $U$.

In this work, we identify a new energy scale associated with charge transfer between regions with different local stacking configurations in the moiré superlattice. When the energy cost of charge transfer $\Delta$ is comparable to or smaller than the local Coulomb repulsion $U$, the MottHubbard description becomes inadequate. Instead, we show that a new type of correlated insulator emerges at half-filling, known as the charge-transfer insulator [18]. Using large-scale first-principles calculation, we obtain the parameters $\Delta$ for various TMD heterobilayers and find that $\Delta$ in $\mathrm{WSe}_{2} / \mathrm{WS}_{2}$ superlattice is comparable to the experimentally observed charge gap [15, 16], whereas $U$ is much larger. We provide a theoretical description of the charge transfer phenomenon by introducing an effective honeycomb lattice model, in which the $M M$ and $M X$ sublattices correspond to "moiré cations" and "moiré anions" where charges are locally concentrated. We note that previous works on twisted bilayer graphene have shown the interaction induced charge redistribution within a local moire region 19 21. Here the charge transfer we predict in TMD moiré superlattices takes place on the length scale of the moiré period $(\sim 10 \mathrm{~nm})$ and can be directly observed by scanning tunneling spectroscopy (STS).

Continuum Model - We consider a heterobilayer TMD such as $\mathrm{WSe}_{2} / \mathrm{WS}_{2}$, with $a\left(a^{\prime}\right)$ as the lattice constant of top (bottom) layer, and $\theta$ as the twist angle. The lattice mismatch leads to a moiré superlattice in Fig. 11, with superlattice constant $L_{\mathrm{M}}=a / \sqrt{\delta^{2}+\theta^{2}}$ where $\delta=(a-$ $\left.a^{\prime}\right) / a^{\prime}$. As illustrated in Fig. 2 b, the valence bands of two layers have a large band offset $\Delta E_{g}$, which is listed for various TMD heterobilayers at zero twist angle in Table. S1. Given the large band offset, the low-energy moiré bands result from the spatial variation of valence band maximum of $\mathrm{WSe}_{2}$ due to the lattice mismatch with $\mathrm{WS}_{2}$ [22], which is described by a long-period moiré potential acting on holes in $\mathrm{WSe}_{2}$. 
(a)
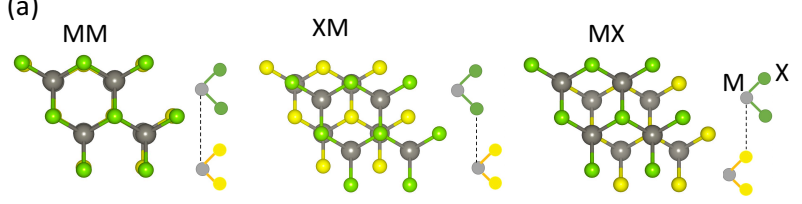

(b)

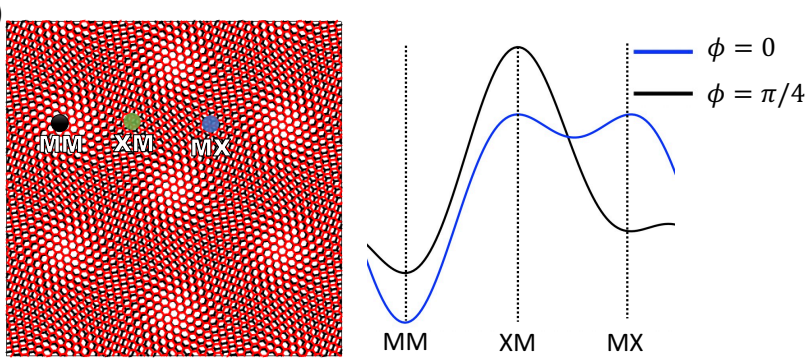

FIG. 1: (a) Lattice structure of MM, MX, XM spots for AA stacking heterobilayer ( $\mathrm{M}$ stands for metal atom and $\mathrm{X}$ stands for chalcogen atom), (b) Real-space moiré pattern of heterobilayer TMD heterobilayer with $\delta=4.0 \%$, where MM, MX, XM spots within one supercell are labeled, and schematic diagram for moiré potential landscape for $\phi=0$ and $\phi=\pi / 4$, along the path from MM to XM and MX spots as indicated by the array in the left figure.

In this work, we study TMD heterobilayers with a small twist angle starting from AA stacking, where the metal atom and chalcogen atom of the top layer are aligned with metal atom and chalcogen atom at the bottom layer, respectively [41]. There are three types of Wykoff positions in a moiré unit cell-hereafter referred to as MM, XM, MX, depending on the alignment of the metal atom $(\mathrm{M})$ and chalcogen atom $(\mathrm{X})$. As shown in Fig. 13, at MM, the metal atoms on top and bottom layers are aligned, While at MX (XM), the metal atom on the top (bottom) layer is aligned with the chalcogen atom on the bottom (top) layer. In the long moiré wavelength limit $L_{\mathrm{M}} / a \rightarrow \infty$, the valence band maximum varies slowly over the moiré unit cell, which can be expressed as the first-order harmonics with moiré wave vectors $\boldsymbol{G}_{i}=\frac{4 \pi}{\sqrt{3}} L_{\mathrm{M}}^{-1}\left(\cos \frac{i 2 \pi}{3}, \sin \frac{i 2 \pi}{3}\right)(\mathrm{i}=1,2,3)[13$.

This is captured by the continuum model $H_{0}=$ $\int \psi^{\dagger}(\boldsymbol{r}) \hat{\mathcal{H}} \psi(\boldsymbol{r}) d^{2} \boldsymbol{r}$ with

$$
\begin{aligned}
\hat{\mathcal{H}} & =-\frac{\nabla^{2}}{2 m}+V(\boldsymbol{r}), \\
V(\boldsymbol{r}) & =-2 V_{0} \sum_{i=1}^{3} \cos \left(\boldsymbol{G}_{i} \cdot \boldsymbol{r}+\phi\right),
\end{aligned}
$$

where $\psi^{\dagger}=\left(\psi_{\uparrow}^{\dagger}, \psi_{\downarrow}^{\dagger}\right)$ creates the holes and $m>0$ is the effective mass. From first principle calculation with the relaxed layer spacing(see Supplemental Material), we find the moiré valence bands within $200 \mathrm{meV}$ are formed by $\pm K$ pockets in $\mathrm{WSe}_{2}$. Owing to strong Ising spin-orbit coupling, valley indices are locked with spin [23, and the two valleys are decoupled at long moiré wavelength limit. $V_{0}>0$ and $\phi$ are the only parameters associated (a)
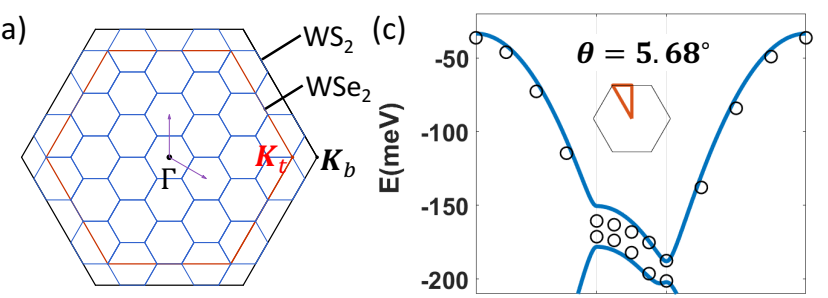

(b)

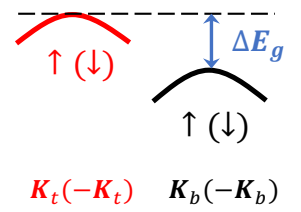

(d)

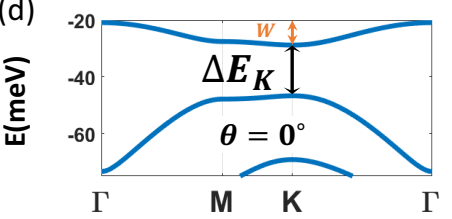

FIG. 2: (a) Brillouin zone (BZ) folding in $\mathrm{WSe}_{2} / \mathrm{WS}_{2}$ moiré superlattice, where $a_{W S e_{2}} / a_{W S_{2}}$ is taken as $6 / 5$ for the illustrative purpose. (b) Schematic low-energy band structure from two layers where $\pm \boldsymbol{K}_{t(b)}$ are two valleys of top (bottom) layer. (c) DFT band structure(open circle) and continuum model band structure (blue lines) at $\theta=5.68^{\circ}$, (d) continuum model band structures of $\mathrm{WSe}_{2} / \mathrm{WS}_{2}$ at $\theta=0^{\circ}$.

\begin{tabular}{cccccc}
\hline \hline System & $\delta$ & $\Delta E_{g}$ & $V_{0}$ & $\phi$ & $E_{0}^{\min }$ \\
\hline $\mathrm{WSe}_{2} / \mathrm{WS}_{2}$ & $4 \%$ & 640 & 15 & $45^{\circ}$ & 1.2 \\
$\mathrm{WSe}_{2} / \mathrm{MoS}_{2}$ & $4 \%$ & 940 & 11 & $40^{\circ}$ & 1.2 \\
$\mathrm{MoSe}_{2} / \mathrm{MoS}_{2}$ & $4 \%$ & 630 & 9 & $42^{\circ}$ & 1.3 \\
$\mathrm{MoSe}_{2} / \mathrm{WS}_{2}$ & $4 \%$ & 270 & 7 & $35^{\circ}$ & 1.3 \\
\hline \hline
\end{tabular}

TABLE I: Summary of heterobilayer TMD. Here $\delta$ is the lattice constant mismatch with respect to the bottom layer, $\Delta E_{g}$ is the band offset, $V_{0}$ and $\phi$ are parameters of moiré potential and $E_{0}^{\min }=\delta^{2} /\left(2 m a^{2}\right)$ is the moiré kinetic energy at zero twist. All energies are in unit of meV.

with the magnitude and overall phase of the three lowest Fourier components of the moiré potential. When the moiré period is large, $V_{0}, \phi$ are intrinsic material properties independent of $L_{\mathrm{M}}$, which we hereafter refer to as moiré potential strength and moiré phase respectively.

To obtain the values of $V_{0}, \phi$, we first use the large-scale density functional theory (DFT) to calculate the moiré band structure of $\mathrm{WSe}_{2} / \mathrm{WS}_{2}, \mathrm{WSe}_{2} / \mathrm{MoS}_{2}$, $\mathrm{MoSe}_{2} / \mathrm{MoS}_{2}$, and $\mathrm{MoSe}_{2} / \mathrm{WS}_{2}$ at the commensurate angel $\theta=5.68^{\circ}$, as shown in Fig. 22. The spinorbit coupling is included via DFT[24, 25] as implemented in the Vienna Ab initio Simulation Package [26]. The interaction between electrons and ionic cores is approximated by the projector augmented wave method, and the exchange-correlation potential was described by the Perdew-Burke-Ernzerhof generalized gradient approximation 27] with the vdW correction incorporated by the vdW-DF (optB86) functionals 28. We assume rigid lattice along with in-plane directions and relax interlayer distance. Depending on the different vdW cor- 


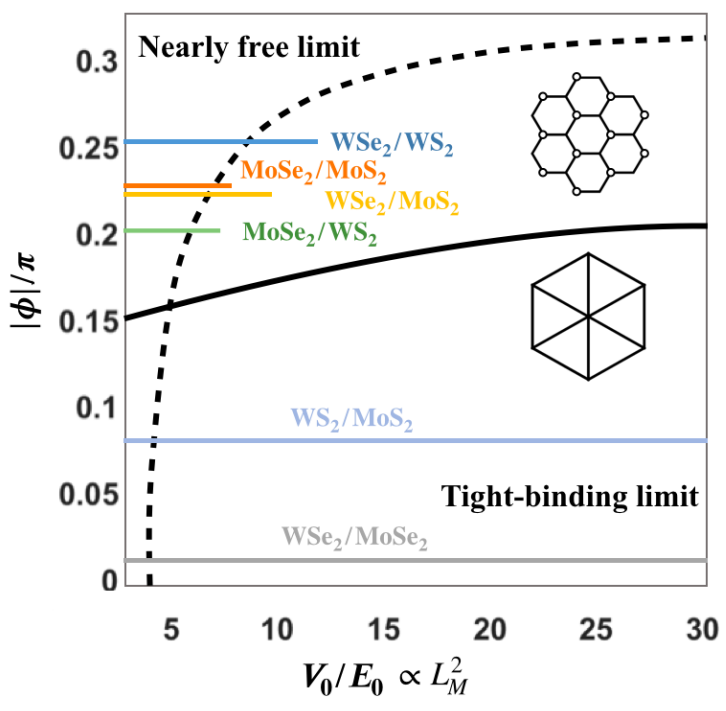

FIG. 3: Systems listed in Table. S1 and $\mathrm{WSe}_{2} / \mathrm{MoSe}_{2}$, $\mathrm{WS}_{2} / \mathrm{MoS}_{2}$ (model parameters in Supplementary Material) can be described by different limits and tight-binding models for the first and second moiré bands. Each colored line denotes a bilayer TMD, and ends at untwisted limit where $V_{0} / E_{0}$ reaches maximum $V_{0} / E_{0}^{\mathrm{min}}$. Nearly free limit and tight-binding limit are separated by the dashed line where $W=\Delta$. Within the tight-binding limit, the tight-binding model is a honeycomb lattice formed by MM and MX (open circle) spots with $s$ orbital on each site above the solid black line, and a triangular lattice formed by MM spots with $s,\left(p_{x}, p_{y}\right)$ orbitals on each site below the solid black line.

rection methods, the interlayer spacing is $6.57 \sim 6.77$ Angstrom. Throughout this range of interlayer distance, we find the moiré band structure is nearly identical.

We find the DFT band structure fits nicely with the continuum model (see Fig. 22), and obtain from this fitting the material specific parameters $V_{0}$ and $\phi$ shown in Table. S1 For $\mathrm{WSe}_{2} / \mathrm{WS}_{2}, V_{0}=15 \mathrm{meV}$ and $\phi=\frac{1}{4} \pi$. Importantly, the moiré phase $\phi$ determines the energy landscape of moiré potential. This can be seen from $V(\boldsymbol{r})$ at three $C_{3}$-symmetric points (Wyckoff positions) $\boldsymbol{r}_{\mathrm{MM}}=\mathbf{0}, \quad \boldsymbol{r}_{\mathrm{MX}}=\frac{1}{\sqrt{3}} L_{\mathrm{M}}(1,0)$ and $\boldsymbol{r}_{\mathrm{XM}}=-\boldsymbol{r}_{\mathrm{MX}}$ respectively. For $0<\phi<\frac{1}{6} \pi$, within one supercell there are one potential minimum (MM) and two maxima (MX and XM), while for $\frac{1}{6} \pi<\phi<\frac{1}{3} \pi$, there are two minima (MM and MX) and one maximum (XM). The four TMD heterobilayers listed in Table. S1. $\mathrm{WSe}_{2} / \mathrm{WS}_{2}$, $\mathrm{WSe}_{2} / \mathrm{MoS}_{2}, \mathrm{MoSe}_{2} / \mathrm{MoS}_{2}$, and $\mathrm{MoSe}_{2} / \mathrm{WS}_{2}$, all belong to the parameter range $\frac{1}{6} \pi<\phi<\frac{1}{3} \pi$, where the presence of two potential minima introduces new physics as we shall show below 42 .

In the following sections, we will study interaction effects in TMD heterobilayers in various regimes of $V_{0}$ and $\phi$. We denote $n_{s}=2$ holes per supercell as the full filling and $n=\frac{1}{2} n_{s}=1$ hole per supercell as the half-filling.

Charge-Transfer Phenomena - In this section, we use Hartree approximation to study the effect of Coulomb interaction on the charge distribution in twisted hetero- bilayer TMD with relatively large bandwidth and demonstrate the charge transfer phenomenon.

The Coulomb interaction including background effect is

$$
H_{C}=\int \delta \rho(\boldsymbol{r}) C\left(\boldsymbol{r}-\boldsymbol{r}^{\prime}\right) \delta \rho\left(\boldsymbol{r}^{\prime}\right) d^{2} \boldsymbol{r} d^{2} \boldsymbol{r}^{\prime}
$$

where $\delta \rho \equiv \psi^{\dagger} \psi-\bar{\rho}$ is the deviation of local hole density from the average $\bar{\rho}$ (which is set by gate voltage), and $C(\boldsymbol{r})=e^{2} /(4 \pi \epsilon|\boldsymbol{r}|)$ is the Coulomb potential with dielectric constant $\epsilon$, which controls the interaction strength. We approximate the Coulomb interaction $H_{C}$ by the mean-field Hatree potential $V_{H}$ self-consistently

$$
V_{H}(\boldsymbol{r})=V(\boldsymbol{r})+\int C\left(\boldsymbol{r}-\boldsymbol{r}^{\prime}\right)\left\langle\delta \rho\left(\boldsymbol{r}^{\prime}\right)\right\rangle d^{2} \boldsymbol{r}^{\prime},
$$

and $\langle\ldots\rangle$ denotes the expectation value in mean-field ground state. As we assume the Hartree potential preserves all symmetries, $V_{H}$ can be written as Fourier series similar to Eq. (2), and the Coulomb interaction only renormalizes the band structure [21, 29].

In Fig. 4a we plot the renormalized filling factor $n / n_{s}$ as a function of chemical potential $\mu$ in $\mathrm{WSe}_{2} / \mathrm{WS}_{2}$ heterobilayer at twist angle $\theta=3^{\circ}$ with different dielectric constants. At low fillings, the charge is always localized at MM spots. As we increase the filling, more holes will be accumulated and the repulsive interaction renormalizes the charge distribution to make it more homogeneous. Near half-filling $n=\frac{1}{2} n_{s}$, when the interaction is weak, the charge distribution remains at MM spots as shown in Fig. 4 $4 \mathrm{~b}$. When the interaction is strong, charge transfer from MM to MX spots occurs and the corresponding charge distribution is shown in Fig. 4k.

In real space, the interaction-induced, fillingdependent charge transfer leads to a significant change of charge distribution on the scale of $10 \mathrm{~nm}$, which can be detected by scanning tunneling spectroscopy (STS). In the energy domain, charge transfer affects band structure on the scale of $10 \mathrm{meV}$, which may be detected in angle-resolved photoemission spectroscopy and optical measurement of the exciton spectrum.

To go beyond the mean-field approximation, in the following we provide a theoretical description of charge transfer physics in TMD heterobilayer with a sufficiently large $L_{\mathrm{M}}$, where the moiré bandwidth $W$ is small compared to the moiré potential $V_{0}$.

Tight-Binding Limit - We first introduce the moiré kinetic energy as $E_{0} \equiv\left(2 m L_{\mathrm{M}}^{2}\right)^{-1}$, which increases with the twist angle as $E_{0} \propto\left(\theta^{2}+\delta^{2}\right)$. When moiré potential is weak compared with kinetic term $V_{0} \ll E_{0}$ (nearly free limit), the first and second bands have a negative indirect gap (e.g. Fig. 2b). When moiré potential is much stronger than kinetic term $V_{0} / E_{0} \gg 1$ (tightbinding limit), moiré bands become flat compared with band gaps $W \ll \Delta$ (e.g. Fig. 2d). All untwisted heterobilayers listed in Table I belong to the tight-binding limit as shown in Fig. 3. 


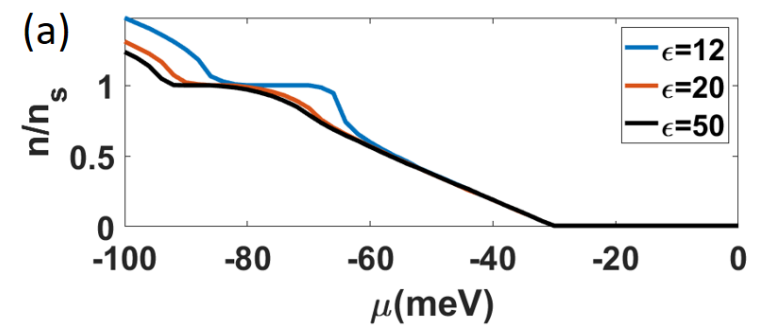

(b)

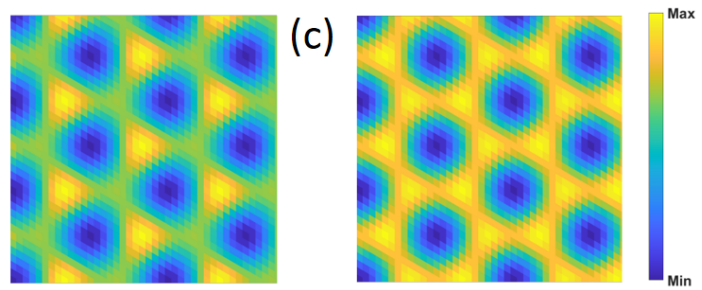

FIG. 4: (a) Filling factor $n / n_{s}$ as a function of chemical potential $\mu$ in $\mathrm{WSe}_{2} / \mathrm{WS}_{2}$ system at $\theta=3^{\circ}$ with $\epsilon=12,20,50$. (b) and (c) are charge distribution at half filling when $\epsilon=50$ and 12 .

In the tight-binding limit, each potential minimum traps a set of local Wannier orbitals. The lowest-energy one is $s$-orbital, and the next is $\left(p_{x}, p_{y}\right)$ doublet. The first moiré band is predominantly formed by $s$ orbitals at MM spots, which are global potential minimum in the parameter range of interest $\phi \in\left(0, \frac{1}{3} \pi\right)$. The character of the second moiré band depends on $\phi$. For $\phi \in\left(0, \frac{1}{6} \pi\right)$, it comes from $p$ orbitals at MM spots. For $\phi \in\left(\frac{1}{6} \pi, \frac{1}{3} \pi\right)$ and in a wide range of $V_{0}$, it comes from $s$-orbitals at MX spots that are local potential minima (see Fig. 22. The energy difference between $s$ orbitals in MX and MM spots defines a charge transfer gap $\Delta_{0}=\varepsilon_{M X}-\varepsilon_{M M}$.

By expanding $V(\boldsymbol{r})$ around a potential minimum, we obtain the characteristic size of $s$-orbitals in MM and MX spots from the harmoinc approximation

$$
\begin{aligned}
& \psi(r, R)=\frac{1}{\sqrt{\pi} \xi} \exp \left(-\frac{|r-R|^{2}}{2 \xi^{2}}\right) \\
& \xi_{M M}=(\cos \phi)^{-1 / 4} \xi_{0}, \xi_{M X}=\left[\sin \left(\phi-\frac{1}{6} \pi\right)\right]^{-1 / 4} \xi_{0} \\
& \xi_{0}=\left(4 \pi^{2} m V_{0}\right)^{-1 / 4} \sqrt{L_{\mathrm{M}}}
\end{aligned}
$$

where $\xi_{M X}$ only applies to $\phi \in\left(\frac{1}{6} \pi, \frac{1}{3} \pi\right)$. It is important to note that for large $L_{\mathrm{M}}, \xi_{i} \propto \sqrt{L_{\mathrm{M}}}$ is parametrically smaller than the moiré period. Therefore, the local Coulomb repulsion is the largest interaction energy, given by

$$
U_{i}=\frac{e^{2}}{4 \sqrt{2 \pi} \epsilon \xi_{i}} \propto L_{\mathrm{M}}^{-1 / 2}
$$

with $i=M M, M X$. In contrast, the interaction between nearest neighbors $V^{\prime}$ is proportional to $1 / L_{\mathrm{M}}$ and hence parametrically smaller than $U$.

In Fig. S3 of the Supplementary Material we plot the bandwidth $W$ of the first moiré band and interaction energies $U, V^{\prime}$ of $\mathrm{WSe}_{2} / \mathrm{WS}_{2}$ at different twist angles. While $U, V^{\prime}$ decrease with $L_{\mathrm{M}}$ in a power-law manner, $W$ is exponentially small in the tight-binding regime. For untwisted $\mathrm{WSe}_{2} / \mathrm{WS}_{2}$, we find $L_{\mathrm{M}}=8.2$ $\mathrm{nm}, \xi_{M M}=2.3 \mathrm{~nm}, \xi_{M X}=3.0 \mathrm{~nm}$ and $U_{M M}=764 / \epsilon$ $\mathrm{meV}, U_{M X}=594 / \epsilon \mathrm{meV}, W=8 \mathrm{meV}, \Delta E_{K}=18 \mathrm{meV}$, $V^{\prime}=302 / \epsilon \mathrm{meV}$.

Depending on the relative strengths of interaction energy, bandwidth, and charge transfer gap, we find three phases at half-filling.

(I) Metal: $U \ll W$. The system is gapless. Under doping, additional charges are mainly localized at MM spots with $s$-orbital symmetry.

(II) Mott insulator: $\Delta>U \gg W$. The insulating ground state has one hole per MM spot, and the charge gap is $U$. When doped further, additional charges are mainly localized around MM spots. In this case, the triangular lattice Hubbard model is a good description 13 .

(III) Charge-transfer insulator: $U>\Delta \gg W$. The insulating ground state has one hole per MM spot, but the charge gap is $\Delta$. When further doped, additional charges are mainly localized at MX spots, thus resulting in charge transfer on moiré scale as the filling increases.

The insulating gap at half-filling inferred from thermal activation of resistivity is only around $10 \mathrm{meV}$ [15, 16], which is significantly smaller than the estimated on-site repulsion $U \sim 128 \mathrm{meV}$ assuming $\epsilon=6$. (Note the distance from the sample to metallic gates is $20 \mathrm{~nm}$ so that screening has little effect on local repulsion $U$ ). However, the measured gap is comparable to the charge transfer gap $\Delta E_{K} \sim 18 \mathrm{meV}$. We thus conclude that the insulating phase at half-filling in untwisted $\mathrm{WSe}_{2} / \mathrm{WS}_{2}$ is likely a charge-transfer insulator, rather than a Mott-Hubbard insulator.

In order to capture the physics of charge transfer between MM and MX spots, we introduce an extended Hubbard model on the honeycomb lattice:

$H=\frac{\Delta}{2} \sum_{i}(-)^{i} c_{i}^{\dagger} c_{i}-t \sum_{\langle i j\rangle}\left(c_{i}^{\dagger} c_{j}+h . c.\right)+\sum_{i j} V_{i j} n_{i} n_{j},(8)$ where $c_{i}=\left\{c_{i \uparrow}, c_{i \downarrow}\right\}^{\mathrm{T}}$ denotes $s$-orbital holes, $(-)^{i}= \pm$ for $i=\mathrm{MX}(\mathrm{MM})$ spots and $t$ denotes hopping. $V_{i j}$ is the Coulomb repulsion between $s$ orbitals at site $i$ and $j$, which includes both on-site repulsion $U_{M M}, U_{M X}$, nearest-neighbor repulsion $V^{\prime}$ and etc. When there is strong screening from the metallic gates, interactions decay rapidly with the distance between sites.

At temperatures below the charge gap, double occupancy is strongly suppressed by the on-site repulsion $U$. For the triangular lattice Hubbard model, the low-energy physics is described by the $t-J$ model[30, 31] with hopping $t$ and antiferromagnetic superexchange interaction $J=4 t^{2} / U>0$ between nearest neighbors. Magnetic susceptibility as a function of doping at various temperatures is shown in Supplementary Material. For chargetransfer insulators such as $\mathrm{WSe}_{2} / \mathrm{WS}_{2}$ described by the honeycomb lattice model (8), their magnetic properties call for future study. 
The extended Hubbard model in honeycomb lattice has also been realized with cold atom in optical lattice 32 36. However, in cold atom systems, the lowest accessible temperature at present is on the order of hopping $t$, which is much higher than the exchange interaction $J$ 37]. In the TMD heterobilayer $\mathrm{WSe}_{2} / \mathrm{WS}_{2}$, the exchange energy is around $J \sim 0.05 \mathrm{meV}$ and the corresponding temperature $(\sim 1 K)$ is readily accessible for experiments.

In conclusion, we present a theory that maps the long period moiré system onto an atomic crystal with cation and anion and studies the correlated insulating behavior. We find that the interplay between moiré potential and interaction strength gives rise to charge transfer insulator in heterobilayer TMD, and opens the possibility of novel electronic states upon doping.

\section{Acknowledgment}

We thank Kin Fai Mak and Jie Shan for sharing their experimental results with us prior to publication, and Hiroki Isobe, Zhen Bi, Taige Wang, and Feng Wang for helpful discussions. This work is supported by DOE Office of Basic Energy Sciences under Award de-sc0018945, N.Y. was partly supported by the U.S. Department of Energy, Office of Science, Basic Energy Sciences, under Award Number de-sc0020149.
[1] Y. Cao, V. Fatemi, A. Demir, S. Fang, S. L. Tomarken, J. Y. Luo, J. D. Sanchez-Yamagishi, K. Watanabe, T. Taniguchi, E. Kaxiras, et al., Nature 556, 80 (2018).

[2] Y. Cao, V. Fatemi, S. Fang, K. Watanabe, T. Taniguchi, E. Kaxiras, and P. Jarillo-Herrero, Nature 556, 43 (2018).

[3] G. Chen, L. Jiang, S. Wu, B. Lyu, H. Li, B. L. Chittari, K. Watanabe, T. Taniguchi, Z. Shi, J. Jung, et al., Nature Physics 15, 237 (2019).

[4] G. Chen, A. L. Sharpe, P. Gallagher, I. T. Rosen, E. J. Fox, L. Jiang, B. Lyu, H. Li, K. Watanabe, T. Taniguchi, et al., Nature 572, 215 (2019), URL https://doi.org/ $10.1038 / \mathrm{s} 41586-019-1393-\mathrm{y}$

[5] R. Bistritzer and A. H. MacDonald, Proceedings of the National Academy of Sciences 108, 12233 (2011).

[6] N. N. Nam and M. Koshino, Physical Review B 96, 075311 (2017).

[7] M. Koshino, N. F. Yuan, T. Koretsune, M. Ochi, K. Kuroki, and L. Fu, Physical Review X 8, 031087 (2018).

[8] S. Carr, S. Fang, Z. Zhu, and E. Kaxiras, Physical Review Research 1, 013001 (2019).

[9] H. Yoo, R. Engelke, S. Carr, S. Fang, K. Zhang, P. Cazeaux, S. H. Sung, R. Hovden, A. W. Tsen, T. Taniguchi, et al., Nature materials 18, 448 (2019).

[10] K. Uchida, S. Furuya, J.-I. Iwata, and A. Oshiyama, Physical Review B 90, 155451 (2014).

[11] M. Van Wijk, A. Schuring, M. Katsnelson, and A. Fasolino, 2D Materials 2, 034010 (2015).

[12] Z. Bi, N. F. Yuan, and L. Fu, arXiv preprint arXiv:1902.10146 (2019).

[13] F. Wu, T. Lovorn, E. Tutuc, and A. H. MacDonald, Physical review letters 121, 026402 (2018).

[14] F. Wu, T. Lovorn, E. Tutuc, I. Martin, and A. MacDonald, Physical review letters 122, 086402 (2019).

[15] Y. Tang, L. Li, T. Li, Y. Xu, S. Liu, K. Barmak, K. Watanabe, T. Taniguchi, A. H. MacDonald, J. Shan, et al., Nature 579, 353 (2020).

[16] E. C. Regan, D. Wang, C. Jin, M. I. B. Utama, B. Gao, X. Wei, S. Zhao, W. Zhao, Z. Zhang, K. Yumigeta, et al., Nature 579, 359 (2020).

[17] J. Hubbard, Proceedings of the Royal Society of London. Series A. Mathematical and Physical Sciences 276, 238 (1963).
[18] J. Zaanen, G. Sawatzky, and J. Allen, Physical Review Letters 55, 418 (1985).

[19] L. Rademaker and P. Mellado, Physical Review B 98, 235158 (2018).

[20] L. Rademaker, D. A. Abanin, and P. Mellado, arXiv preprint arXiv:1907.00940 (2019).

[21] F. Guinea and N. R. Walet, Proceedings of the National Academy of Sciences 115, 13174 (2018).

[22] C. Zhang, C.-P. Chuu, X. Ren, M.-Y. Li, L.-J. Li, C. Jin, M.-Y. Chou, and C.-K. Shih, Science advances 3, e1601459 (2017).

[23] D. Xiao, G.-B. Liu, W. Feng, X. Xu, and W. Yao, Physical review letters 108, 196802 (2012).

[24] M. H. Naik and M. Jain, Physical review letters 121, 266401 (2018).

[25] L. Xian, M. Claassen, D. Kiese, M. M. Scherer, S. Trebst, D. M. Kennes, and A. Rubio, arXiv preprint arXiv:2004.02964 (2020).

[26] G. Kresse and J. Furthmüller, Computational materials science 6, 15 (1996).

[27] J. P. Perdew, K. Burke, and M. Ernzerhof, Physical review letters 77, 3865 (1996).

[28] J. Klimeš, D. R. Bowler, and A. Michaelides, Physical Review B 83, 195131 (2011).

[29] T. Cea, N. R. Waler, and F. Guinea, arXiv preprint arXiv:1906.10570 (2019).

[30] J. Spalek, arXiv preprint arXiv:0706.4236 (2007).

[31] P. A. Lee, N. Nagaosa, and X.-G. Wen, Reviews of modern physics 78, 17 (2006).

[32] D. Jaksch, C. Bruder, J. I. Cirac, C. W. Gardiner, and P. Zoller, Physical Review Letters 81, 3108 (1998).

[33] W. Hofstetter, J. I. Cirac, P. Zoller, E. Demler, and M. Lukin, Physical review letters 89, 220407 (2002).

[34] M. Greiner, O. Mandel, T. Esslinger, T. W. Hänsch, and I. Bloch, nature 415, 39 (2002).

[35] N. Gemelke, X. Zhang, C.-L. Hung, and C. Chin, Nature 460, 995 (2009).

[36] P. Soltan-Panahi, J. Struck, P. Hauke, A. Bick, W. Plenkers, G. Meineke, C. Becker, P. Windpassinger, M. Lewenstein, and K. Sengstock, Nature Physics 7, 434 (2011).

[37] A. Mazurenko, C. S. Chiu, G. Ji, M. F. Parsons, M. Kanász-Nagy, R. Schmidt, F. Grusdt, E. Demler, D. Greif, and M. Greiner, Nature 545, 462 (2017). 
[38] N. Mounet, M. Gibertini, P. Schwaller, D. Campi, A. Merkys, A. Marrazzo, T. Sohier, I. E. Castelli, A. Cepellotti, G. Pizzi, et al., Nature nanotechnology 13, 246 (2018).

[39] B. Bauer, L. Carr, H. G. Evertz, A. Feiguin, J. Freire, S. Fuchs, L. Gamper, J. Gukelberger, E. Gull, S. Guertler, et al., Journal of Statistical Mechanics: Theory and Experiment 2011, P05001 (2011).

[40] T. Koretsune and M. Ogata, Physical review letters 89, 116401 (2002).

[41] Alternatively, $\mathrm{AB}$ stacking can be viewed as a $180^{\circ}$ rotation of top layer

[42] $\mathrm{WS}_{2} / \mathrm{MoS}_{2}$ and $\mathrm{WSe}_{2} / \mathrm{MoSe}_{2}$ are discussed in Supplementary material.

\section{Appendix A: Commensurate structure}

The lattice constants for $\mathrm{MoS}_{2}, \mathrm{MoSe}_{2}, \mathrm{WS}_{2}$ and $\mathrm{WSe}_{2}$ are 3.18818, 3.31579, 3.18719 and 3.31698 Angstrom respectively 38 . For bilayer with the same chalcogen atoms, the lattice mismatch is less than $0.1 \%$, While for bilayer with different chalcogen atoms, the lattice mismatch is around $4 \%$. To build a commensurate structure from two different monolayers with same type Bravais lattices, we consider a bilayer system, whose primitive vectors are denoted by $\left\{\boldsymbol{a}_{1}, \boldsymbol{a}_{2}\right\}$ and $\left\{\boldsymbol{a}_{1}^{\prime}, \boldsymbol{a}_{2}^{\prime}\right\}$ respectively. When the bilayer system is a commensurate superlattice with primitive vectors $\left\{\boldsymbol{L}_{1}, \boldsymbol{L}_{2}\right\}$, we have 13

$$
\begin{aligned}
& \left(\begin{array}{l}
\boldsymbol{L}_{1} \\
\boldsymbol{L}_{2}
\end{array}\right)=M\left(\begin{array}{l}
\boldsymbol{a}_{1} \\
\boldsymbol{a}_{2}
\end{array}\right)=M^{\prime}\left(\begin{array}{l}
\boldsymbol{a}_{1}^{\prime} \\
\boldsymbol{a}_{2}^{\prime}
\end{array}\right), \\
& M=\left(\begin{array}{cc}
m & n \\
p & q
\end{array}\right), \quad M^{\prime}=\left(\begin{array}{cc}
m^{\prime} & n^{\prime} \\
p^{\prime} & q^{\prime}
\end{array}\right),
\end{aligned}
$$

where $m, n, p, q, m^{\prime}, n^{\prime}, p^{\prime}, q^{\prime}$ are eight integers. These integers are determined by lattice mismatch between two layers (i.e. twist angle and strain) and also information of each layer (such as the anisotropy ratio). The most general way to determine these integers out of lattice information is through enumeration. We search all eight integers in a given range and compute corresponding lattice information for every given set of eight integers. When the calculated lattice information matches with the given one (up to some given precision), we then find the solution. In the following, however, we will discuss two special classes of superlattices where these eight integers have analytical solutions. When the two monolayers with threefold rotations, i.e. they are all triangular lattices, the integer matrices $M, M^{\prime}$ have to be conformal (i.e. a scalar times a rotation). Without loss of generality, let us assume $\boldsymbol{a}_{1,2}=a(1 / 2, \pm \sqrt{3} / 2)$ and $\boldsymbol{a}_{1,2}^{\prime}=a^{\prime} R(\theta)(1 / 2, \pm \sqrt{3} / 2)$ with $a^{\prime} \geqslant a$, namely the unprimed layer is not rotated while the primed layer is rotated by angle $\theta$ along out-of-plane direction. Then the conformal matrices $M, M^{\prime}$ will have the following form

$$
\begin{gathered}
M=N\left(\begin{array}{cc}
\cos \phi-\frac{1}{\sqrt{3}} \sin \phi & -\frac{2}{\sqrt{3}} \sin \phi \\
\frac{2}{\sqrt{3}} \sin \phi & \cos \phi+\frac{1}{\sqrt{3}} \sin \phi
\end{array}\right), \\
M^{\prime}=N^{\prime}\left(\begin{array}{cc}
\cos \phi^{\prime}-\frac{1}{\sqrt{3}} \sin \phi^{\prime} & -\frac{2}{\sqrt{3}} \sin \phi^{\prime} \\
\frac{2}{\sqrt{3}} \sin \phi^{\prime} & \cos \phi+\frac{1}{\sqrt{3}} \sin \phi^{\prime}
\end{array}\right),
\end{gathered}
$$

where $N, N^{\prime}$ are positive integers and $\phi, \phi^{\prime}$ are angles. Since each one of $M, M^{\prime}$ is effectively described by two parameters, we can write

$$
M=\left(\begin{array}{cc}
m & n \\
-n & m-n
\end{array}\right), \quad M^{\prime}=\left(\begin{array}{cc}
m^{\prime} & n^{\prime} \\
-n^{\prime} & m^{\prime}-n^{\prime}
\end{array}\right) .
$$

Furthermore, we want to consider first-order moiré pattern for simplicity, which is defined in terms of reciprocal vectors. Assume the reciprocal lattice vectors of two layers and the superlattice are $\left\{\boldsymbol{G}_{1}, \boldsymbol{G}_{2}\right\},\left\{\boldsymbol{G}_{1}^{\prime}, \boldsymbol{G}_{2}^{\prime}\right\}$ and 
$\left\{\boldsymbol{g}_{1}, \boldsymbol{g}_{2}\right\}$ respectively, then the first-order moiré pattern is defined by the conditions

$$
\boldsymbol{g}_{i}=\boldsymbol{G}_{i}-\boldsymbol{G}_{i}^{\prime}
$$

With this condition, it is found that $m-m^{\prime}=n-n^{\prime}=$ 1 , and hence the twist angle and lattice constant ratio between two layers are

$$
\begin{array}{r}
\theta=\arctan \left(\frac{\sqrt{3}(m-n)}{2\left(m^{2}+n^{2}-m n\right)-m-n}\right), \\
r \equiv \frac{a^{\prime}}{a}=\sqrt{\frac{m^{2}+n^{2}-m n}{m^{2}+n^{2}-m n-m-n+1}}
\end{array}
$$

and the superlattice vectors are

$$
\begin{array}{r}
\boldsymbol{L}_{1}=m \boldsymbol{a}_{1}+n \boldsymbol{a}_{2}, \quad \boldsymbol{L}_{2}=-n \boldsymbol{a}_{1}+(m-n) \boldsymbol{a}_{2}, \\
L \equiv\left|\boldsymbol{L}_{i}\right|=\frac{a}{\sqrt{1-2 r \cos \theta+r^{2}}} .
\end{array}
$$

Here for TMDs with less than $0.1 \%$ lattice mismatch, we construct the commensurate moiré superlattice via twist the top layer with angles $\theta=$ $21.78^{\circ}, 13.1^{\circ}, 9.43^{\circ}, 6.59^{\circ}$ and $5.49^{\circ}$. While for TMDs with lattice mismatch around $4 \%$, the calculated twist angels are $\theta=16.31^{\circ}, 7.31^{\circ}, 6.39^{\circ}, 5.68^{\circ}$ and $4.72^{\circ}$.

\section{Appendix B: Details of the ab-initio calculation and parameter fitting}

For $\mathrm{MoSe}_{2} / \mathrm{WSe}_{2}$ and $\mathrm{MoS}_{2} / \mathrm{WS}_{2}$ with nearly identical lattice constant, we fit the continue model parameters from the energy shift of band maxima from the relative shift of monolayer unit cells 13, as shown in Table. S1. We note that the fitting from large-scale DFT as we have done in the main text would give different parameters, and leave the more realistic treatment such as lattice relaxation at the long moiré wavelength limit to future study.

For another four systems with lattice mismatch $4 \%$, we fit the parameters of periodic potential and its phase factor directly from DFT band structures at various commensurate structures with different twist angles, a further calculation of DFT charge density distribution is performed to fix sign of the phase factor. We note that bilayer structures with twist angle $\theta=5.68^{\circ}$ fall into the gauge that $K$ pockets of monolayer unit cell fold to $\Gamma$ point of moiré BZ. The band structures of various TMD heterobilayers with unequal monolayer lattice constants are summarized in Fig. S1. Note the energy of $\Gamma$ pockets in $\mathrm{MoSe}_{2}$ is only lower by $120 \mathrm{meV}$ compared with $\mathrm{K}$ pockets in $\mathrm{MoSe}_{2}$, which gives rise to the relatively flat second Moiré band around $\Gamma$ point in moiré BZ in Fig. $\mathrm{S} 1$ ( $\mathrm{a}, \mathrm{b})$.

Since the charge transfer physics is dependent on the bandwidth $W$ and charge transfer gap $\Delta$, we choose the
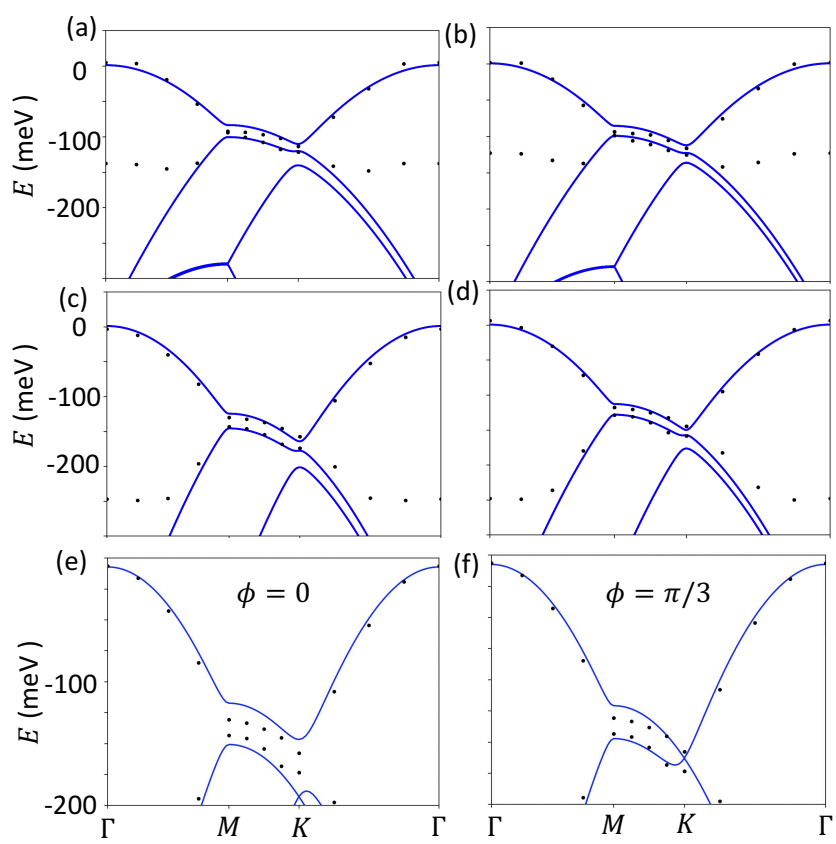

FIG. S1: Continuum model fitting of DFT band structure at twist angle $\theta=5.68^{\circ}$ of (a) $\mathrm{MoSe}_{2} / \mathrm{MoS}_{2}$, (b) $\mathrm{MoSe}_{2} / \mathrm{WS}_{2}$, (c) $\mathrm{WSe}_{2} / \mathrm{MoS}_{2}$ and (d) $\mathrm{WSe}_{2} / \mathrm{WS}_{2}$. DFT band structure of $\mathrm{WSe}_{2} / \mathrm{WS}_{2}$ at twist angle $\theta=5.68^{\circ}$ and continuum model fitting with $V=15 \mathrm{meV}$ and (e) $\phi=0$, (f) $\phi=\pi / 3$.

\begin{tabular}{cccccc}
\hline \hline System & $\delta$ & $\Delta E_{g}$ & $V_{0}$ & $\phi$ & $E_{0}^{\min }$ \\
\hline $\mathrm{WSe}_{2} / \mathrm{MoSe}_{2}$ & $<0.1 \%$ & 370 & 8.14 & $-4^{\circ}$ & $<10^{-3}$ \\
$\mathrm{WS}_{2} / \mathrm{MoS}_{2}$ & $<0.1 \%$ & 360 & 6.52 & $-13^{\circ}$ & $<10^{-3}$ \\
\hline \hline
\end{tabular}

TABLE S1: Summary of heterobilayer TMD. Here $\delta$ is the lattice constant mismatch with respect to the bottom layer, $\Delta E_{g}$ is the band offset, $V_{0}$ and $\phi$ are parameters of moiré potential and $E_{0}^{\min }=\delta^{2} /\left(2 m a^{2}\right)$ is the moiré kinetic energy at zero twist. All energies are in unit of $\mathrm{meV}$.

bandwidth of the first moiré band and the bandgap between first and second moiré band as the essential criteria for the fitting of continuum model parameters. As expected, the potential strength $V_{0}$ determines the moiré bandwidth, while the potential phase factor $\phi$ determines the bandgap at $K$ point in the moiré BZ. As shown in Fig. [S1, we also list the band structure fitting with different phase factor $\phi$ for $\mathrm{WSe}_{2} / \mathrm{WS}_{2}$ heterobilayer, which shows a large bandgap in $K$ at $\phi=0$ and a degenerate $K$ point at $\phi=\pi / 3$ for the first and second moiré band.

\section{Appendix C: Fock term and Details of charge transfer}

The full mean-field treatment of the Coulomb interaction also includes Fock decomposition $\delta \rho(\boldsymbol{r}) \delta \rho\left(\boldsymbol{r}^{\prime}\right) \rightarrow$ $\psi^{\dagger}(\boldsymbol{r}) \psi\left(\boldsymbol{r}^{\prime}\right)\left\langle\psi\left(\boldsymbol{r}^{\prime}\right) \psi^{\dagger}(\boldsymbol{r})\right\rangle$, which results in the mean- 
field Hamiltonian $H_{\mathrm{MF}}=\int \psi^{\dagger}(\boldsymbol{r}) g\left(\boldsymbol{r}-\boldsymbol{r}^{\prime}\right) \psi\left(\boldsymbol{r}^{\prime}\right) d^{2} \boldsymbol{r} d^{2} \boldsymbol{r}^{\prime}$ with renormalized propagator $g(\boldsymbol{r})=g_{0}(\boldsymbol{r})+$ $C(\boldsymbol{r})\left\langle\psi(\mathbf{0}) \psi^{\dagger}(\boldsymbol{r})\right\rangle$, where $g_{0}(\boldsymbol{r})$ is the bare propagator. We expect that the moiré potential and hence charge transfer physics discussed in this work would not be affected by Fock term too much.

We can introduce a dimensionless quantity to describe the charge imbalance between MM and MX spots

$$
P=\frac{n_{\mathrm{MM}}-n_{\mathrm{MX}}}{n_{\mathrm{MM}}+n_{\mathrm{MX}}},
$$

and plot it as a function of chemical potential $\mu$ together with filling factor, as shown in Fig. S2a and b. As we can see, at low filling, the charge is mainly concentrated at MM spots $P>0$, when doping increases, MM and MX spots first become balanced in terms of charge distribution $P \rightarrow 0$, and then MX spots can have more charges than MM spots $P<0$ since repulsion at MX spots is weaker than MM. As explicit examples, we also plot charge distribution at different fillings $n=0.5 n_{s}$ and $n=0.1 n_{s}$ in Fig. S2 c and d respectively.
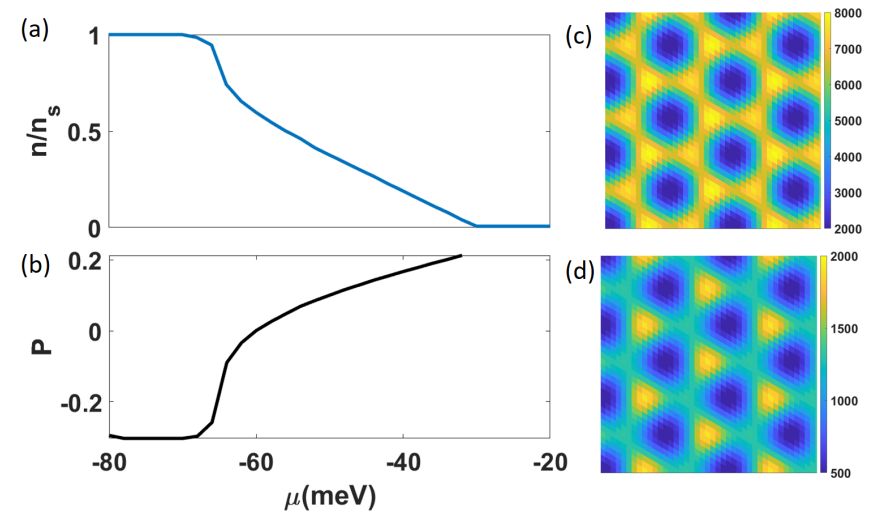

FIG. S2: Charge transfer in $\mathrm{WSe}_{2} / \mathrm{WS}_{2}$ system at twist angle $\theta=3^{\circ}$ with dielectric constants $\epsilon=12$. (a) and (b) are filling factor $n / n_{s}$ and charge imbalance parameter $P$ as functions of chemical potential $\mu,(\mathrm{c})$ and (d) are charge distribution at different fillings $n=0.5 n_{s}$ and $n=0.1 n_{s}$ respectively. Units of colorbars are arbitrary.

\section{Appendix D: Band structures, Coulomb interactions, and tight-binding models}

In Fig. S3 we plot onsite repulsion $U$, nearestneighbor repulsion $V^{\prime}$ and bandwidth $W$ of $\mathrm{WSe}_{2} / \mathrm{WS}_{2}$ at different twist angles. All three energy scales decrease with decreasing angle $\theta$ (or equivalently increasing moiré wavelength $L_{\mathrm{M}}$ ), but $W$ decreases faster than interactions.

As shown in Fig. 2, there are two regimes in the phase space spanned by moiré potential strength $V_{0} / E_{0}$ and moiré phase $\phi$, where the second moiré bands are qualitatively different.
Triangular regime where the second and third moiré bands are from $\left(p_{x}, p_{y}\right)$ orbitals at MM spots and the corresponding tight-binding model is

$$
H_{\triangleright}=\sum_{i} c_{i}^{\dagger} \hat{\Delta} c_{i}-\sum_{\langle i j\rangle}\left(c_{i}^{\dagger} \hat{T} c_{j}+h . c .\right),
$$

where $\boldsymbol{c}=\left\{s, p_{x}, p_{y}\right\}^{\mathrm{T}}$ denotes $s$ - and $p_{x, y}$-orbital holes, and $i$ is MM spot. $\hat{\Delta}=\operatorname{diag}\left(\varepsilon_{s}, \varepsilon_{p}, \varepsilon_{p}\right)$ denotes onsite energy matrix, where $\varepsilon_{s}, \varepsilon_{p}$ denote the energy of $s$ and $\left(p_{x}, p_{y}\right)$ orbitals at MM spot respectively.

Honeycomb regime where the second moiré band is from $s$ orbitals at MX spots and the corresponding tightbinding model is

$$
H_{\square}=\frac{\Delta}{2} \sum_{i}(-)^{i} c_{i}^{\dagger} c_{i}-t \sum_{\langle i j\rangle}\left(c_{i}^{\dagger} c_{j}+h . c .\right),
$$

where $c_{i}=\left\{c_{i \uparrow}, c_{i \downarrow}\right\}^{\mathrm{T}}$ denotes $s$-orbital holes, $(-)^{i}= \pm$ for $i=\mathrm{MX}(\mathrm{MM})$ spots and $t$ denotes hopping. And $\Delta=\varepsilon_{M X}-\varepsilon_{M M}$ denotes the charge-transfer gap from MM to MX spots.

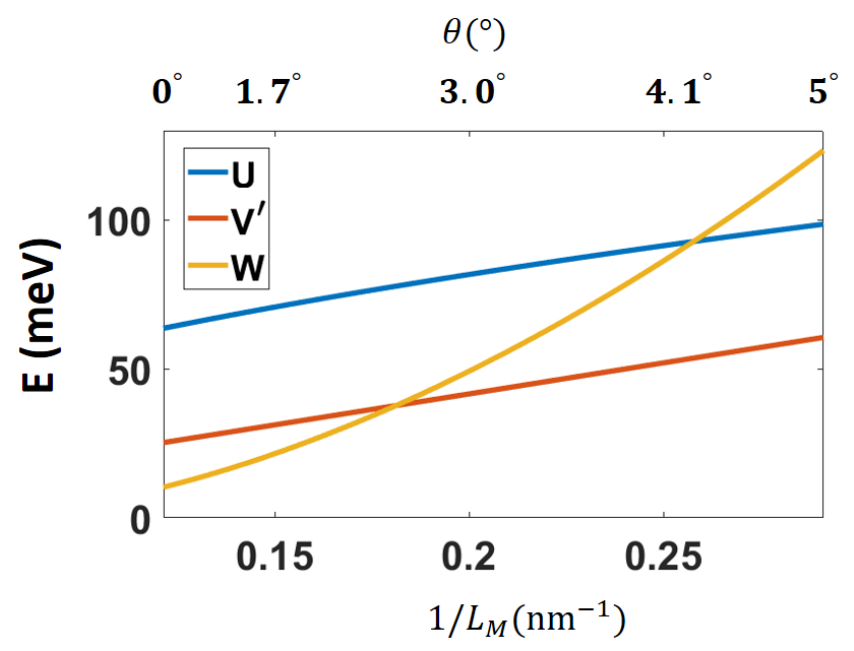

FIG. S3: Onsite Coulomb repulsion $U$, nearest-neighbor repulsion $V^{\prime}$ and bandwidth $W$ of $\mathrm{WSe}_{2} / \mathrm{WS}_{2}$ as functions of moiré wavelength $L_{\mathrm{M}}$ (bottom axis) and twist angle $\theta$ (top axis) when dielectric constant is chosen as $\epsilon=12$.

\section{Appendix E: Magnetic Properties}

We also study the magnetic properties of $t$ - $J$ model in the triangular lattice. We performed ED (exact diagonalization) 39] on the triangular lattice $t-J$ model, and calculate temperature and filling dependent spin susceptibility.

From the Curie-Weiss law plot of susceptibilities in Fig. S4 inset, we found that Curie temperature $T_{0}$ moved towards zero when we increased the filling factor higher than half, indicating that antiferromagnetic correlation 
is reduced by doping. As shown in Fig. S4, in a wide temperature range, spin susceptibility is a non-monotonic function of filling factor $\nu=n / n_{s}$ with a maximal peak at optimal filling $\nu(T)$. Among them, at higher temperature $T \gg t$ the peak locates exactly at the half-filling $\nu=\frac{1}{2}$, while at lower temperature $T \lesssim t$, the susceptibility peak is shifted to above half-filling $\nu>\frac{1}{2}$, in agreement with the result from high-temperature expansion study 40 . These findings are consistent with the spin susceptibility of $\mathrm{WSe}_{2} / \mathrm{WS}_{2}$ heterobilayers inferred from optical spectroscopy under the magnetic field [15].

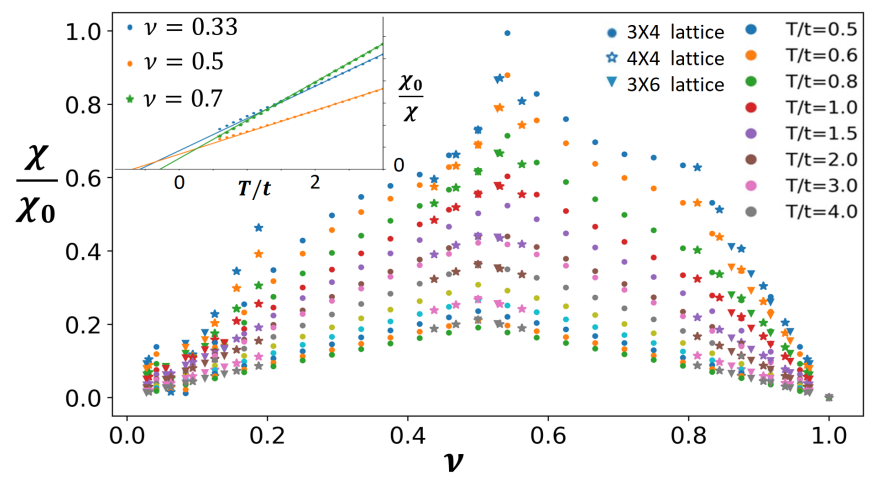

FIG. S4: Uniform spin susceptibility of $t$ - $J$ model at $J / t=0.4$ $(U / t=10)$. The peak is around $\nu=n / n_{s}=\frac{13}{24}$ for $0.5 t<T<1.5 t$, but at $\nu=\frac{1}{2}$ for $T>1.5 t$. Here $\chi_{0}=t^{-1}$, and $t$ is around $1 \mathrm{meV}$. Inset: Inverse spin susceptibility as a function of temperature, where dots are numerical results and solid lines are Curie-Weiss fit. The Curie temperature $T_{0}$ is largest in magnitude at half-filling and gradually moves towards zero as the doping deviates from half-filling, indicating the transition from AFM to PM. 